\title{
Autores de agressão sexual em contextos intra e extrafamiliar: revisão da literatura
}

\author{
* Lucilene Paiva Costa \\ ** Lília Chaves Cavalcante \\ *** Daniela Castro Reis
}

\begin{abstract}
Resumo
Este estudo objetivou identificar semelhanças e diferenças nas características biopsicossociais de autores de agressão sexual de crianças e/ou adolescentes no contexto intrafamiliar e extrafamiliar, a partir de estudos publicados entre 1983 e 2013. Realizou-se a busca em base de dados eletrônica - Portal Periódicos da Coordenação de Aperfeiçoamento de Pessoal de Nível Superior empíricos, nos idiomas inglês e português. Foram encontradas 1.122 publicações, sendo selecionadas 64 para leitura integral por três juízes, sendo que 56 artigos atenderam aos critérios de inclusão, 43 tratavam simultaneamente do contexto intrafamiliar e extrafamiliar. Deste total, selecionou-se 21 artigos por conterem dados da caracterização da população estudada, distinguindo-as por contexto de ocorrência intrafamiliar e extrafamiliar. Estudos desta natureza podem auxiliar na compreensão do que pode conduzir uma pessoa a praticar agressão sexual contra crianças e/ou adolescentes e os aspectos contextuais de suas trajetórias de vida.
\end{abstract}

Palavras-chave: autores de agressão sexual; crianças e adolescentes; contexto intrafamiliar e extrafamiliar.

\section{Authors of Sexual Aggression in Context Intra and extrafamilial: Literature Review}

\begin{abstract}
This study aimed to identify similarities and differences in the biopsychosocial characteristics of authors of sexual aggression of children and / or adolescents in the intrafamily and extrafamilial context, based on studies published between 1983 and 2013. The search was conducted in an electronic database - Portal Periódicos da Coordenação de Aperfeiçoamento de Pessoal de Nível Superior, with reading of the title and summary of the complete and empirical studies, in the English and Portuguese languages. A total of 1.122 publications were found, of which 64 were selected for full reading by three judges, of these 56 articles met the inclusion criteria, 43 dealt simultaneously with the intrafamilial and extrafamilial context. Of this total, 21 articles because they contain data of the characterization of the study population, distinguishing them by intrafamily and extrafamiliar occurrence context. From this total, 21 articles were selected because they contain data on the characterization of the study population, distinguishing them by intrafamily and extrafamilial context. Studies of this nature may help in understanding what can lead a person to engage in sexual assault against children and / or adolescents and the contextual aspects of their life trajectories.
\end{abstract} Key words: authors of sexual assault; children and adolescents; intrafamily and extrafamiliar contexts.

* Psicóloga e Mestre pela UFPA. Pedagoga pela UFPA e analista na Defensoria Pública do Estado do Pará na area de pedagogia.

** Mestre em Serviço Social e Doutora pelo Programa de Pós-Graduação em Teoria e Pesquisa do Comportamento da Universidade Federal do Pará. Pós-Graduação em Psicologia pela Universidade Federal do Rio Grande do Sul. Professora do curso de serviço Social da UFPA.

*** Psicóloga pela Universidade da Amazônia. Doutorado e Mestrado em Psicologia pela UFPA. Professora efetiva da Universidade Federal Rural da Amazônia. 


\section{Introdução}

Estudos de Revisão de Literatura (RL) possuem como principal objetivo agregar evidências de pesquisas. O uso desses estudos como fonte de evidências para organizar várias publicações respondendo a uma pergunta de pesquisa formulada de forma clara, vem crescendo no meio acadêmico. Utiliza-se revisão da literatura como uma forma metodológica de buscar conhecer e compreender um tema já estudado, identificando lacunas, semelhanças, diferenças e inconsistências entre os estudos de uma determinada problemática de pesquisa (Galvão, Pansani, \& Harrad, 2015; Vosgerau \& Romanowski, 2014).

Este estudo trata-se de uma revisão da literatura de produções científicas empíricas, nacionais e internacionais, referentes à agressão sexual cometida por adultos contra crianças e adolescentes em contextos intrafamiliar e extrafamiliar, com foco em características biopsicossociais dos autores que a praticaram. Este estudo de revisão diferencia-se de outros realizados anteriormente, em âmbito nacional e internacional, por incluir não apenas estudos referentes a características sociodemográficas, mas também características psicológicas da população estudada.

A produção sobre autores de agressão sexual é recente, principalmente em âmbito nacional. Os estudos de Hohendorff, Habigzang e Koller (2012) e Souto, Lucena, Paiva e Cavalcanti (2010) abordaram a temática que envolve autores de agressão sexual e suas vítimas. O objetivo desses estudos referiu-se principalmente à análise do perfil dos envolvidos neste tipo de agressão e a descrição de suas características sociodemográficas.

O estudo sobre violência sexual contra meninos realizado por Hohendorff, Habigzang e Koller (2012), evidenciou que autores de agressão sexual de meninos, em geral, foram caracterizados como heterossexuais do sexo masculino. Esse estudo evidenciou também que meninos com menos de seis anos estariam mais expostos à agressão sexual no contexto intrafamiliar, e, entre aqueles acima de doze anos, o risco para essa agressão seria maior no contexto extrafamiliar. No estudo de revisão sistemática da literatura de Souto, Lucena, Paiva e Cavalcanti (2010), constatou-se que os autores de agressão sexual eram em sua maioria do sexo masculino e que poucas publicações analisadas tratavam de aspectos específicos relacionados ao autor de agressão sexual, ocorrendo mais a associação da agressão sexual com fatores de risco.

Em âmbito internacional o estudo de revisão sistemática da literatura de Marotta (2015) investigou a avaliação de formas de tratamento e o estudo de Tully, Chou e Browne (2013) a reincidência do comportamento de agressão sexual. Esses estudos trataram da temática que envolve autores de agressão sexual e suas vítimas.
Marotta (2015) revisou artigos sobre avaliação de programas destinados a tratar autores de agressão sexual com deficiência intelectual e desabilidades desenvolvimentais. Este estudo demonstrou que os tratamentos cognitivo-comportamentais têm sido a modalidade mais comum, utilizada entre autores de agressão sexual que possuem deficiência intelectual e desabilidades desenvolvimentais.

No estudo de Tully, Chou e Browne (2013), investigou-se a eficácia dos instrumentos de avaliação de risco na previsão de reincidência de autores de agressão sexual. Entre os resultados dessa revisão, enfatizou-se o fato de todos os instrumentos apresentavam moderada acurácia preditiva. Ou seja, estes não poderiam ser considerados precisos na avaliação dos riscos para o comportamento de reincidência da população em que foram aplicados.

O presente estudo de revisão da literatura teve como objetivo analisar as características biopsicossociais de autores de agressão sexual de crianças e/ou adolescentes no contexto intrafamiliar e extrafamiliar presentes nas produções nacional e internacional, publicadas entre 1983 a 2013, buscando resposta para a questão: quais as diferenças e semelhanças entre as características biopsicossociais de autores de agressão sexual de crianças e adolescentes dos contextos intrafamiliar e extrafamiliar? Em termos teóricos-metodológicos avaliou-se as características da população estudada utilizando como referencial o modelo bioecológico de Urie Bonfrenbrenner, que leva em consideração o contexto ecológico em que a pessoa está inserida (Bronfenbrenner, 2011).

\section{Método}

\section{Procedimentos Gerais}

Esta revisão da literatura foi adequada ao Modelo de Principais Itens para Relatar Revisões sistemáticas e Meta-análises (PRISMA), o qual compreende as etapas de escolha das fontes de dados, seleção dos descritores, busca aos artigos, análise dos títulos e resumos, leitura dos textos na íntegra, adoção de critérios de inclusão e exclusão, extração dos dados e avaliação dos artigos.

Para a realização do levantamento sistemático dos artigos publicados entre os anos de 1983 a 2013 foi utilizado Portal Periódico da Coordenação de Aperfeiçoamento de Pessoal de Nível Superior (CAPES). Os descritores utilizados nas buscas aos artigos foram: agressor sexual, abusador sexual, perpetrador sexual, molestador sexual e estuprador, assim como seus correspondentes no idioma inglês, os quais foram combinados com as palavras criança/child e adolescente/adolescent.

Para a seleção da produção científica foram utilizados os seguintes critérios de inclusão: publicação nos 
idiomas português e inglês, entre o período de 1983 a 2013; artigos completos e empíricos, voltados para estudos com população de autores de agressão sexual adultos de criança e adolescente. Foram excluídos os artigos que contemplaram as seguintes situações: literatura cinzenta (documentação técnica, anúncio de jornais, monografias, dissertações, teses); estudos teóricos e de revisão da literatura, investigações sobre sexualidade e/ou doenças sexualmente transmissíveis, estudos que avaliaram somente vítimas de agressão sexual; pesquisas com animais; artigos com as seguintes tipologias: pedófilo, psicopata, aliciador, traficante sexual e explorador sexual.

Após a seleção preliminar, os artigos foram submetidos a uma nova seleção realizada por três pesquisadores, os quais atuaram como juízes, de maneira independente. Esses juízes aplicaram um teste de relevância com o ponto de corte 3 . Ou seja, cada artigo para ser selecionado deveria receber resposta positiva em três das quatro questões contidas no teste de relevância aplicado. Deste modo, a permanência do artigo para a análise ocorreu quando houve concordância de dois juízes.

Após o a aplicação do teste de relevância foi realizado o cálculo do Índice de Confiabilidade (IC), por meio do número de concordâncias e discordâncias entre os juízes. O cálculo do IC é uma técnica de pesquisa que demonstra a probabilidade do resultado de uma pesquisa ter credibilidade e isso ocorre quando o resultado do IC é $\geq 80 \%$. Neste estudo, o IC foi de $87.50 \%$. Este índice foi calculado por meio da equação: $\mathrm{IC}=\mathrm{A}$ (concordância) $\mathrm{x} 100 \div \mathrm{A}+\mathrm{D}$ (discordância) $/ \mathrm{IC}=56 \times 100 \div 56+$ $8=87.50 \%$ (Pereira, 2006).

\section{Procedimentos de análise}

Os artigos selecionados $(\mathrm{N}=56)$ foram analisados em duas etapas: primeiro ocorreu uma caracterização geral, onde se organizou a categorização geral dos artigos de acordo com os dados referentes à publicação, à agressão em estudo, à população estudada e ao método de pesquisa. Na segunda etapa, optou-se por analisar de forma mais específica às características da agressão sexual, assim como as características biopsicossociais dos autores da agressão sexual contidas nos artigos.

\section{Resultados}

Nas buscas pelos artigos foram encontradas 1.122. Após a leitura dos títulos e resumos selecionaram-se 64 estudos nos quais foram aplicados os critérios de inclusão e exclusão pela leitura dos textos na íntegra, resultando em um total de 56 artigos submetidos ao Teste de Relevância entre os juízes.

Entre os 56 artigos selecionados $77 \%$ tratavam simultaneamente do contex to intrafamiliar e extrafamiliar,
$14 \%$ referiam-se somente ao contexto intrafamiliar, $4 \%$ somente ao contexto extrafamiliar e não foi informado o contexto de ocorrências das agressões sexuais em 5\% dos artigos.

Do total das publicações que tratavam simultaneamente do contexto intrafamiliar e extrafamiliar $(n=43)$, analisaram-se 21, pois somente estas publicações demonstraram em seus resultados a caracterização da população estudada de acordo com o contexto de ocorrência intrafamiliar e extrafamiliar. (Veja figura 1).

As amostras dos artigos selecionados eram compostas, principalmente, por pessoas do sexo masculino (93\%), em sua maioria condenados que estavam cumprindo pena em prisões (84\%), entre estes, $3,5 \%$ eram clérigos antes do encarceramento. Foram encontrados diferentes tipos de vínculos entre os autores de agressão sexual e suas vítimas. No contexto intrafamiliar, identificou-se vínculo de parentesco entre os autores de agressão sexual e as vítimas sendo, principalmente, pai biológico ou padrasto $(38.8 \%)$. No contexto extrafamiliar, verificou-se de forma predominante pessoas desconhecidas para as vítimas (85\%).

Entre as características da agressão sexual referente ao contexto intrafamiliar, constatou-se: o longo período de tempo (meses, anos); ocorrência em locais em que os autores possuíam grande quantidade de controle, como suas residências e locais de trabalho e menor reincidência dos autores da agressão sexual quando realizavam tratamento. No contexto extrafamiliar nas características da agressão em estudo identificou-se: curto período de tempo (menos de uma semana, única vez); ocorrência na residência dos autores, em veículos e em locais que as vítimas costumavam frequentar como escolas, parques e praças. Nos dois contextos estudados as vítimas, eram predominantemente, do sexo feminino.

Sobre as semelhanças entre as características biopsicossociais do autor da agressão sexual contidas nos artigos analisados, no que se refere à característica biológica identificou-se, principalmente, a preferência por vítimas do sexo feminino. Em relação às semelhanças psicológicas, averiguou-se no estudo de Sanfelice e De Antoni, (2010) não haver diferença entre os autores de agressão sexual dos contextos estudados na escala Psychopathy Checklist Revised - PCL-R, que caracterizou os participantes do estudo pela superficialidade, falsidade, insensibilidade, crueldade, ausência de afeto, remorso e culpa. Nas semelhanças referentes às características sociais, identificou-se exposição frequente à violência na mídia durante a infância, laços de apego inseguro com os pais (Simons, Wurtele \& Durham, 2008) e não possuir noção clara sobre o significado do termo sexualidade (Sanfelice \& De Antoni (2010). 
Figura 1. Fluxograma de seleção dos artigos da revisão, segundo recomendações PRISMA.

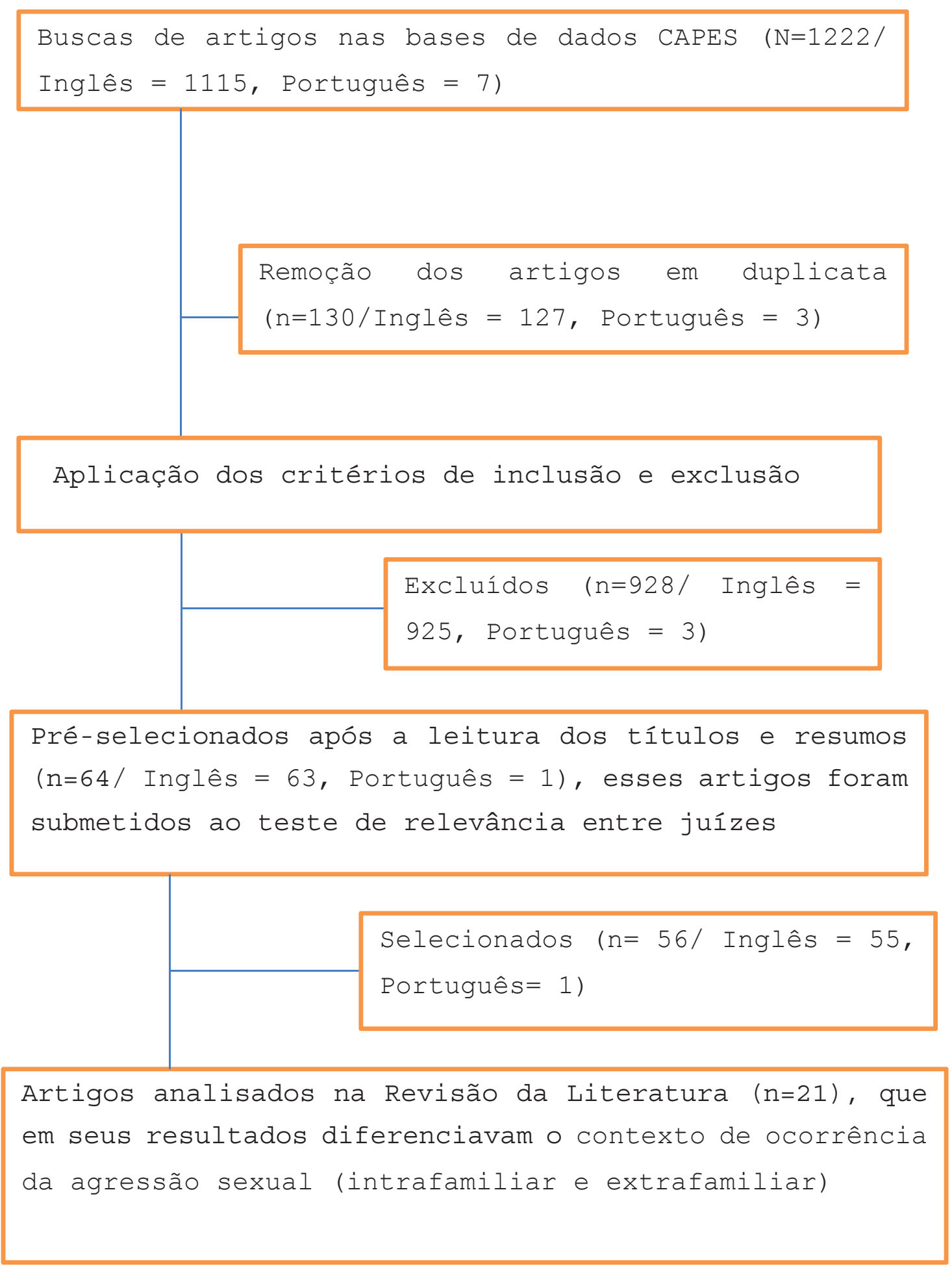


Nas diferenças identificadas entre as características biopsicossociais da população estudada, no que se refere ao aspecto biológico, constatou-se que no contexto intrafamiliar os autores de agressão sexual foram identificados como mais velho do que aqueles do contexto extrafamiliar, (Lussier, Bouchard, \& Beauregard, 2011; Rebocho \& Gonçalves, 2012; Rice \& Harris, 2002. Também se constataram diferenças entre as características psicológicas.

Tabela 1. Diferenças entre as Características Psicológicas dos Autores de Agressão Sexual (Seção de resultados).

\begin{tabular}{ll|l}
\hline \multicolumn{2}{l}{ Autores das publicações } & \multicolumn{2}{l}{ Contextos } \\
\cline { 2 - 3 } & Intrafamiliar & extrafamiliar \\
\hline Mussier, Bouchard e Beauregard (2011), & $\begin{array}{l}\text { Bem sucedidos na estratégia de manter } \\
\text { vítimas únicas que eram repetidamente } \\
\text { revitimizadas }\end{array}$ & $\begin{array}{l}\text { Bem sucedidos na estratégia de completar as } \\
\text { agressões sexuais em múltiplas vítimas, em geral } \\
\text { uma agressão por vítima. }\end{array}$ \\
\hline Titcomb, Delahunty e Puiseau (2012) & $\begin{array}{l}\text { Maior probabilidade de aceitar } \\
\text { tratamento referente ao crime sexual. }\end{array}$ & $\begin{array}{l}\text { Menor probabilidade de aceitar tratamento } \\
\text { referente ao crime sexual. }\end{array}$ \\
\hline Rebocho e Gonçalves (2012) & $\begin{array}{l}\text { Mais prováveis de possuir } \\
\text { características de comportamento do } \\
\text { modus operandi manipulador. }\end{array}$ & $\begin{array}{l}\text { Mais prováveis de possuir características de } \\
\text { comportamento do modus operandi coercitivo. }\end{array}$ \\
\hline Carvalho e Nobre (2013) & $\begin{array}{l}\text { Maior afeto positivo, sensação de } \\
\text { alegria, estado de alerta, energia, } \\
\text { entusiasmo e autoconfiança. }\end{array}$ & $\begin{array}{l}\text { Maior afeto negativo, irritabilidade, angústia e } \\
\text { hostilidade. }\end{array}$ \\
\hline Elilott e Kilcoyne (1995) & $\begin{array}{l}\text { Utilização de persuasão e suborno } \\
\text { para assegurar a continuidade das } \\
\text { agressões. }\end{array}$ & $\begin{array}{l}\text { Utilização de força física e armas para assegurar } \\
\text { a continuidade das agressões. }\end{array}$ \\
\hline
\end{tabular}

Quanto às diferenças referentes ao âmbito social identificou-se entre os autores de agressão sexual de crianças e adolescentes no contexto intrafamiliar: baixo risco para reincidência (Mailloux, Abracen, Serin, Cousineau, Malcolm \& Looman, 2003); quando eram autores de agressão de vítimas de ambos os sexos tiveram o maior percentual de reincidência (Langevin \& Curnoe, 2012); mais ajustamento social e comportamento sexual menos desviante (Rice \& Harris, 2002); menos condutas antissociais e menos condenações por crimes violentos (Mailloux et al, 2003); menos prováveis de cometer outros crimes além dos crimes sexuais (Tewksbury, Jennings e Zgoba (2012); relataram mais experiências de abuso sexual na infância e menos experiências de agressão física (Simons, Wurtele, \& Durham, 2008; Tardif, Auclair, Jacob, \& Carpentier, 2005) e também indicaram maior variedade de experiências heterossexuais quando não eram clérigos (Haywood, Kravitz, Grossman, Wasyliw, \& Hardy, 1996; Haywood, Kravitz, Wasyliw, Goldberg, \& Cavanaugh, 1996). No contexto extrafamiliar identificou-se o oposto dessas características de âmbito social.

Analisando os dados da literatura revisada, constatou-se que no contexto intrafamiliar os autores da agressão em estudo possuíam características de comportamento do modus operandi manipulador, eles utilizavam persuasão e suborno para assegurar a continuidade das agressões e relataram mais experiências de abuso sexual na infância. Diferenciando-se do contexto extrafamiliar, em que a população estudada possuía características de comportamento do modus operandi coercitivo, pois utilizavam força física e armas para assegurar a continuidade das agressões e relataram mais experiências de agressão física na infância.

\section{Discussão}

Neste estudo verificou-se que as publicações sobre autores de agressão sexual de crianças e adolescentes em âmbito nacional ainda são escassas, uma vez que o interesse em estudar esta temática é relativamente recente no Brasil. Segundo Esber (2008) no âmbito nacional, o interesse em estudar esse tema surgiu no meio acadêmico em meados dos anos de 1990. Contudo, Ferrari e Vecina (2002) afirmam que os estudos desse tema no Brasil tiveram início a partir da década de 1980. No âmbito internacional, o interesse em estudar os autores de agressão sexual de crianças e adolescentes teve início nas décadas de 1950 e 1960, com os estudos de Ellis, Doorbar e Johnston (1954) e de Cowen e Strickler (1963).

No total de artigos encontrados nas buscas obteve-se um número elevado de publicações relacionadas às vítimas de agressão sexual (225), o que correspondeu a $24 \%$ de publicações descartadas, este dado confirma que 
os estudos referentes à agressão sexual contra crianças e adolescentes ainda se concentram, principalmente, nas vítimas. Os motivos que levam à existência de elevado número de estudos que focalizam a vítima em detrimento do autor da agressão sexual são diversos. Entre eles, destaca-se a dificuldade em conseguir amostras para realização das pesquisas. Neste estudo de revisão da literatura observou-se que as amostras dos artigos eram compostas, predominantemente, por condenados presos, este fato justifica-se por se tratar de uma população de difícil acesso.

Os participantes dos estudos selecionados eram, predominantemente, do sexo masculino, entre os 21 artigos selecionados nesta revisão, somente um estudo possuía amostra com pessoas do gênero feminino. De acordo com Burn e Brown (2005), existem evidências empíricas de que a agressão sexual infanto-juvenil é praticada de forma predominante por homens, independente do contexto onde ocorre e do sexo das vítimas agredidas. Segundo Sattler (2011) mulheres também praticam agressão sexual, porém em menor proporção que os homens.

Presume-se que, independente do sexo da pessoa que pratica agressão sexual contra crianças e adolescentes, é importante obter informações sobre seu o percurso desenvolvimental, pois essas informações são essenciais para compreender as características que foram desenvolvidas por essas pessoas em suas interações com os diversos ambientes dos quais participou de forma ativa, influenciando e sendo influenciado (Bronfenbrenner, 2011). Do mesmo modo, informações sobre o vínculo entre os autores de agressão sexual e as vítimas, tempo de duração e o local em que praticam essas agressões são importantes, pois estas informações associadas a outras mais retrospectivas, como exposição a fatores de risco na infância, ocorrências de psicopatologias, envolvimento em outras práticas criminais, comportamentos considerados estereotipados, crenças e valores, podem contribuir com a elaboração do perfil dos autores deste tipo de agressão.

Nos estudos revisados identificaram-se ligações importantes entre os autores de agressão sexual e vítimas e os locais onde ocorreram essas agressões. No contexto intrafamiliar a agressão sexual teve longo período de tempo, houve maior ocorrência nos casos em que esses autores eram pai ou padrastos, que praticavam a agressão sexual no ambiente doméstico. No contexto extrafamiliar a agressão sexual teve curto período de tempo, as maiores ocorrências encontradas referiam-se, principalmente a pessoas desconhecidas para às vítimas, essas pessoas praticavam a agressão em diversos locais, entre eles, ruas, praças, parques, veículos e escolas. Com essa informação é possível afirmar que se faz necessário políticas de proteção à família no contexto intrafamiliar e políticas protetivas no contexto extrafamiliar referente aos espaços públicos e instituições educativas.

Os estudos de Titcomb, Delahunty e Puiseau (2012) e Haywood, Kravitz, Grossman, Wasyliw e Hardy (1996) indicaram que às vítimas no contexto intrafamiliar eram mais jovens (crianças), diferente daquelas do contexto extrafamiliar, que eram mais velhas (adolescentes). Quanto ao sexo das vítimas observou-se que na maioria das publicações analisadas (81\%) pertenciam aos sexos feminino e masculino, porém tanto no contexto intrafamiliar quanto no extrafamiliar elas eram predominantemente do sexo feminino. Ou seja, apesar da presença de vítimas de ambos os sexos, o número de vítimas do sexo masculino apresentados era sempre menor em relação ao sexo feminino.

A menor quantidade de vítimas do sexo masculino nos estudos selecionados pode estar relacionada aos estereótipos sexuais presentes nas sociedades, que podem levar crianças e adolescentes do sexo masculino a considerar que a manutenção de relações sexuais com os adultos, especialmente com mulheres, seja uma forma de demonstrar masculinidade. As questões de gênero ainda são marcadas pelo patriarcalismo que gera uma cultura diferenciada para homens e mulheres operando de forma diferente para cada gênero. Existem cobranças sociais em relação ao sexo masculino que carregam estereótipos sobre os papéis que devem desempenhar socialmente. Além disso, pode existir entre o sexo masculino o medo do rótulo da homossexualidade (Stoltenborgh, IJzendoorn, Euser, \& Kranenburg, 2011).

De acordo com os resultados obtidos neste estudo, supõe-se que, diferente dos autores de agressão sexual do contexto extrafamiliar, a maioria das pessoas que se tornaram autoras de agressão sexual no contexto intrafamiliar, desenvolveu-se dentro dos padrões estabelecidos socialmente, considerados aceitáveis, uma vez que desempenhavam suas atividades (tarefas) e diferentes papéis sociais (mãe, pai, padrasto, irmão), por meio de interações sociais consideradas satisfatórias. Também presume-se que as características biopsicossociais desenvolvidas por essas pessoas durante o seu percurso desenvolvimental influenciaram o seu comportamento de praticar agressão sexual.

As características psicológicas identificadas nas publicações analisadas permitiram concluir que, no contexto intrafamiliar, os autores da agressão em estudo premeditavam a agressão, sendo manipuladores e persuasivos com as 
vítimas. Deste modo, eram bem sucedidos na estratégia de manter vítimas únicas, que eram repetidamente revitimizadas, diferenciando-se dos autores de agressão sexual do contexto extrafamiliar, os quais foram identificados como mais agressivos, pois houve a utilização de força física e armas para assegurar a ocorrência do ato sexual, manifestando pouca preocupação em responder pelas consequências desse ato criminoso (Elilott, Browne \& Kilcoyne, 1995; Lussier, Bouchard, \& Beauregard, 2011; Mair, 1993).

Sobre as características sociais vivenciadas pelo público alvo deste estudo, identificou-se que sofrer agressão sexual na infância foi mais relacionado com aqueles que praticaram agressão sexual no contexto intrafamiliar e sofrer agressões físicas foi mais relacionado a aqueles que praticaram essa agressão no contexto extrafamiliar (Simons, Wurtele, \& Durham, 2008; Tardif, Auclair, Jacob, \& Carpentier, 2005).

Levando em consideração as características biopsicossociais identificadas entre os autores de agressão sexual do contexto extrafamiliar nos estudos analisados, observou-se que a maioria dessas pessoas, desenvolveu-se de maneira considerada fora dos padrões estabelecidos socialmente. De acordo com os estudos analisados, essas pessoas frequentemente foram consideradas menos ajustadas socialmente, mais violentas com as vítimas, além de apresentar mais condenações por crimes violentos de alto risco, mais problemas com drogas e mais condutas antissociais, e serem consideradas de alto risco para reincidência da agressão sexual(Rice \& Harris, 2002; Mailloux et al., 2003).

Então, no contexto extrafamiliar,os autores de agressão sexual contra crianças e adolescentes, nas publicações analisadas, demonstraram que não desempenhavam atividades e papéis sociais de modo satisfatório nos diversos ambientes em que estabeleciam interações sociais (Jeglic, Mercado, \& Levenson, 2012; Rice \& Harris, 2002; Mailloux et al., 2003; Tewksbury, Jennings, \& Zgoba, 2012). Presume-se, que as características desenvolvidas por essas pessoas durante o seu percurso desenvolvimental as conduziram a utilizar a coerção e outras formas de violência com frequência. A possibilidade de ter sofrido agressão física na infância, por um período de tempo prolongado, pode ter contribuido para que os autores de agressão sexual de crianças e adolescentes do contexto extrafamiliar reproduzissem esse tipo de comportamento com suas vítimas.

Acredita-se que os conhecimentos produzidos neste estudo de revisão podem contribuir com a elaboração de hipóteses explicativas sobre como ocorreu o processo de desenvolvimento dos autores de agressão sexual nos con- textos estudados. Esses conhecimentos também podem apontar novos questionamentos para realização de outras pesquisas. Mais estudos sobre a temática estudada, ainda precisam ser elaborados, para o melhor conhecimento do perfil dessa população estudada.

Partindo do pressuposto de que as características biopsicossociais, são produtoras e produtos de relações estabelecidas com outras em dado contexto, considera-se que ninguém nasce autor de agressão sexual, mas a pessoa pode tornar-se um, em função de suas características desenvolvidas nas interações estabelecidas nos ambientes em que atuaram e também por diversos fatores, entre eles, fatores de risco, que são condições ambientais ou situações não favoráveis para o desenvolvimento.

\section{Considerações Finais}

As investigações científicas possibilitam, além de novas descobertas, a maior visibilidade dos temas pesquisados, principalmente quando estes são cercados de preconceitos e tabus, que é o caso daqueles que tratam de questões de cunho sexual. No decorrer da realização deste estudo foi possível perceber a necessidade de mais pesquisas científicas sobre a população estudada, principalmente, nacionais, devido à escassez de dados.

Neste estudo verificou-se em diversas publicações, que não havia a informação sobre o contexto de ocorrência dessa agressão, assim como também não havia informações sobre os desdobramentos legais e as consequências ocorridas no ciclo de vida dos autores de agressão sexual após a condenação. Futuras investigações podem obter essas informações, permitindo a revelação de fatores ambientais e comportamentais necessários à compreensão da ocorrência da agressão sexual contra crianças e adolescentes, seja no contexto intrafamiliar ou extrafamiliar.

As publicações internacionais predominaram neste estudo, então os resultados encontrados nesta revisão da literatura seriam encontrados em âmbito nacional em estudos empíricos? Somente a realização de mais pesquisas científicas nacionais possibilitará comparações com a literatura internacional. Outro questionamento pertinente é: o fato de os estudos serem realizados com encarcerados poderia comprometer sua confiabilidade? Visto que, a maioria dos participantes das amostras negou a autoria da agressão sexual e culpou outras pessoas pelo fato de estarem presos, inclusive as próprias vítimas.

Obter conhecimentos sobre o perfil dos autores de agressão sexual de crianças e adolescentes dos contextos, 
intrafamiliar e extrafamiliar, é uma importante contribuição social, uma vez que esses conhecimentos podem possibilitar a elaboração de programas específicos de intervenção e até mesmo de tratamento para essa população. Esses conhecimentos também são importantes porque podem auxiliar profissionais de diversas áreas de conhecimento (educação, jurídica, social) em suas atuações profissionais, no atendimento às famílias com suspeita ou confirmação de ocorrência de agressão sexual.

Neste estudo, a utilização do Modelo Bioecológico do Desenvolvimento Humano, como referencial teórico na análise das publicações permitiu compreender o desenvolvimento dos autores de agressão sexual do contexto intrafamiliar e extrafamiliar, por meio das características biopsicossociais desenvolvidas em suas trajetórias de vida. Deste modo, é possível afirmar que os processos desenvolvimentais da população estudada sofreram variações, em função do conjunto de suas características biopsicossociais e dos ambientes em que estiveram inseridos, direta e indiretamente, no decorrer do tempo.

\section{Referências}

Bronfenbrenner, U. (2011). Bioecologia do desenvolvimento humano: Tornando os seres humanos mais humanos. Porto Alegre: Artmed.

Burn, M.F., \& Brown, S. (2005). A review of cognitive distortions in child sex offenders: An examination of the motivations and mechanisms that underlie the justification for abuse. Aggression and Violent Behavior. Vol. 11. pp. 225-236. Retrieved From: http://www.sciencedirect.com/science/article/pii/ S1359178905000595

Cowen, E. L., \& Strickler, G. (1963). The social desirability of trait descriptive terms: a sample of sexual offenders. The Journal of Social Psychology, v. 59, n. 2, p. 307-315, 1963. Retrieved From: http://www.tandfonline.com/doi/abs/10 .1080/00224545.1963.9919436\#

Ellis, A., Doorbar, R. R \& Johnston, R. (1954). Characteristics of convicted sex offenders. The Journal of Social Psychology. Vol. 40, N. 1, p. 3-15, 1954. Retrieved from: http://www.researchgate.net/publication/254348290_Characteristics_of_Convicted_Sex_Offenders

Elliott, M., Browne, K., \& Kilcoyne, J. (1995, May). Child sexual abuse prevention: What offenders tell us. Child Abuse \& Neglect, Vol. 19(5), pp. 579-594. Retrieved from http://www.sciencedirect.com/science/article/ pii/0145213495000173

Esber, K. M. (2008). Autores de violência sexual contra crianças e adolescentes: um estudo a partir da teoria sócio-histórica. (Dissertação). Universidade Católica Goiânia. Retrieved from: http://tede.biblioteca.ucg.br/tde_arquivos/11/ TDE-2008-07-18T063119Z467/Publico/Karen\%20Michel\%20Esber.pdf

Ferrari, D. C. A., \& Vecina, T. C. C (2002). O fim do silêncio na violência familiar: teoria e prática. São Paulo: Editora Ágora.

Galvão, T. F., Pansani, T. D. S. A., \& Harrad, D. (2015). Principais itens para relatar Revisões sistemáticas e Meta-análises: A recomendação PRIS MA. Epidemiologia e Serviços de Saúde, 24, 335-342. doi: 10.5123/S167949742015000200017

Haywood, L. Grossman., \& J. Cavanaugh. (1990, September). Subjective versus objective measurements of deviant sexual arousal in clinical evaluations of alleged child molesters. Psychological Assessment: A Journal of Consulting and Clinical Psychology, Vol. 2(3), pp.269-275. DOI: 10.1037/1040-3590.2.3.269

Haywood, T. W., Kravitz, H.M., Grossman, L. S., Wasyliw, O. E., \& Hardy, D. W. (1996, June). Psychological aspects of sexual functioning among cleric and noncleric alleged sex offenders. Child Abuse \& Neglect, Vol. 20(6), pp. 527-
536. Retrieved from http://www.sciencedirect.com/science/article/ pii/0145213496000348

Haywood, T.W., Kravitz, H. M., Wasyliw, O. E., Goldberg, J., \& Cavanaugh, J. L. (1996, December). Cycle of abuse and psychopathology in cleric and noncleric molesters of children and adolescents. Child Abuse \& Neglect, Vol. 20(12), pp. 1233-1243. Retrieved from http://www.ncbi.nlm.nih.gov/pubmed/8985613

Hohendorff, J. V., Habigzang, L. F., \& Koller, S. H. (2012). Violência sexual contra meninos: dados epidemiológicos, características e consequências. Psicologia: USP, S.P. 23(2), pp. 395-415. Retrieved From: http://www. scielo.br/pdf/pusp/v23n2/aop0712.pdf

Jeglic, E. L., Mercado, C.C., \& Levenson, J. S. (2012). The prevalence and correlates of depression and hopelessness among sex offenders subject to community notification and residence restriction legislation. Am J Crim Just, 37(1), pp. $46-59$. DOI: $10.1007 /$ s12103-010-9096-9

Langevin, R., \& Curnoe, S. (2011, August). Lifetime criminal history of sex offenders seen for psychological assessment in five decades. International Journal of Offender Therapy and Comparative Criminology, Vol.56(7), pp. 997 - 1021. DOI: 10.1177/0306624X11420084

Lussier, P., Bouchard, M., \& Beauregard, E. (2011, October). Patterns of criminal achievement in sexual offending: Unravelling the "successful" sex ofender. Journal of Criminal Justice, Vol. 39 (5), pp. 433 - 444. DOI:10.1016/j. jcrimjus.2011.08.001

Mailloux, D. L., Abracen, J., Serin, R., Cousineau, C., Malcolm, B., \& Looman, J. (2003, April). Dosage of treatment to sexual offenders: Are we overprescribing? International Journal of Offender Therapy and Comparative Criminology, Vol. 47(2), pp. 171-184. DOI: 10.1177/0306624X03251096

Mair, K. J. (1993). The nature of the act: A neglected dimension in the classification of sex offenders. British Journal of Criminology, Vol. 33(2), pp. 267-275. Retrieved from http://bjc.oxfordjournals.org/content/33/2/267.full.pdf

Marotta, P. L. (2015). A Systematic Review of Behavioral Health Interventions for Sex Offenders with Intellectual Disabilities. Sexual Abuse: A Journal of Research and Treatment 1 - 38. DOI: 10.1177/1079063215569546

Pereira, A. L. (2006). Revisão sistemática da literatura sobre produtos usados no tratamento de feridas. (Dissertação, Universidade Federal de Goiás, Goiânia). Retrieved From: http://repositorio.bc.ufg.br/tede/bitstream/tde/732/1/ Angela\%20Lima\%20Pereira.pdf

Rebocho, M. F., \& Gonçalves, R. A. (2012, April). Sexual predators and prey: A comparative study of the bunting behavior of rapists and child molesters. Journal of Interpersonal Violence, Vol. 27(14), pp. 2770-2789. DOI: $10.1177 / 0886260512438280$

Rice, M. E., \& Harris, G. T. (2002). Men who molest their sexually immature daughters: Is a special explanation required? Journal of Abnormal Psychology, Vol. 111(2), pp. 329-339. DOI: 10.1037/0021-843X.111.2.329

Sanfelice, M. M., \& De Antoni, C. (2010). A percepção do abusador sexual sobre a (sua) sexualidade. Revista Interamericana de Psicologia/Interamerican Journal of Psychology, Vol. 44(1), pp. 131-139. Retrieved From: http:// www.redalyc.org/articulo.oa?id $=28420640014$

Sattler, M. K. (2011). O abusador: o que sabemos. IN: Azambuja., M.R. F \& Ferreira., M. H. M. Violência Sexual contra crianças e adolescentes. Porto Alegre: Artmed.

Simons, D. A., Sandy K. Wurtele, S. K., \& Durham, R. L. (2008, May). Developmental experiences of child sexual abusers and rapists. Child Abuse \& Neglect, Vol. 32(5), pp. 549-560. DOI: 10.1016/j.chiabu.2007.03.027

Souto, R.Q., Lucena, B., Paiva, A. Z., \& Cavalcanti, A. L. (2010). Violência Sexual em Crianças e Adolescentes: Uma Revisão Sistemática. Revista de Ciências Médicas e Biológicas. No. 9 (2): pp. 172-178. Retrieved From: http://www. portalseer.ufba.br/index.php/cmbio/article/view/4953/3614

Stoltenborgh, M., IJzendoorn, M.H., Euser, E.M \& Kranenburg, M. J. (2011). A Global Perspective on Child Sexual Abuse: Meta-Analysis of Prevalence Around the World. Child Maltreatment16 (2) 79 - 101. Retrieved From: http://www. ncbi.nlm.nih.gov/pubmed/21511741

Tardif, M., Auclair, N., Jacob, M., \& Carpentier, J. (2005, February). Sexual abuse perpetrated by adult and juvenile females: An ultimate attempt to resolve a conflict associated with maternal identity. Child Abuse \& Neglect, Vol. 29(2), pp.153-167. DOI: 10.1016/j.chiabu.2004.05.006

Tewksbury, R., Jennings, W. G., \& Zgoba, K. M. (2012). A longitudinal examination of sex ofender recidivism prior to and following the implementation of SORN. Behavioral Sciences and the Law, Vol. 30(3), pp. 308-328. DOI: 10.1002/ bsl.1009 
Titcomb, C., Delahunt, J.G., \& Puiseau, B. W. (2012). Pretrial diversion for intrafamilial child sexual offending: Does biological paternity matter? Criminal Justice and Behavior, Vol. 39(4), pp. 552-570. DOI: 10.1177/0093854811433678

Tully, R. J., Chou, S., \& Browne, K. (2013). A systematic review on the effectiveness of sex offender risk assessment tools in predicting sexual recidivism of adult male sex offenders. Clinical Psychology Review. 33. Pp. 287-316. http://dx.DOI. org/10.1016/j.cpr.2012.12.002
Vosgerau, D. S. R., \& Romanowski. J. P. (2014). Estudos de revisão: implicações conceituais e metodológicas. Rev. Diálogo Educ., Curitiba. V. 14, n. 41, p. $165-$ 189. DOI: $10.7213 /$ dialogo.educ.14.041.DS08

Submetido em: 21-9-2017

Aceito em: 11-12-2018 